\title{
Forum ophthalmicum - how to take control over accumulation of diagnostic data from macular OCT with benefit for both doctor and patient?
}

\section{Dorota Maria Kaczmarek}

Clinical Ophthalmology Center Spektrum, Wroclaw Head: Prof. Maria Hanna Niżankowska, MD, PhD

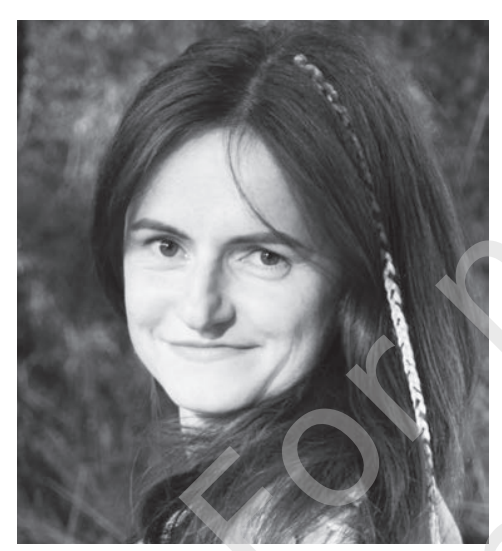

\section{H I G H LI G HTS}

A precise and fast tool for research analysis, the Forum ${ }^{\oplus}$ software, significantly accelerates the diagnostics of macular diseases, which optimizes doctor's working time and facilitates communication with a patient.

\section{ABSTRACT}

Wet age-related macular degeneration is a disease which requires regular diagnostic tests such as macular optical coherent tomography which is extremely important both in diagnosis and disease monitoring. With constant aging of the population, we are dealing with an increasing incidence rate of AMD, which makes it even more important to have a tool allowing for quick and precise analysis of anatomical changes in macular region, particularly in the current complicated epidemiological situation when diagnostic time should be used most efficiently to plan the follow-up treatment. Forum ${ }^{\circ}$ software allows for the precise analysis of many tests performed in a single patient and combining it with treatment effectiveness assessment which substantially accelerates the diagnostics.

Key words: wAMD, optical coherent tomography, anti-VEGF therapy, medical data analysis 


\section{INTRODUCTION}

Age-related macular degeneration (AMD) is a progressive chronic disorder that affects the macula and central retina. It is the leading cause of blindness among people over 55 years and currently affects $8.7 \%$ of the world population [1]. There are two kinds of AMD: dry and wet. The most common form of AMD is dry AMD, of which $85-90 \%$ of AMD patients are diagnosed. Although wet AMD is less common (diagnosed in 10-15\% of patients with AMD), it is associated with a higher percentage of severe visual impairment. Wet AMD is responsible for $80 \%$ of all AMD-related blindness [2-5]. The classification of wet and dry AMD is based on the presence or absence of subretinal/intraretinal abnormal blood vessels referred to as macular neovascularization (MNV) [6].

Three anti-VEGF agents are registered for the treatment of wet AMD (wAMD) in Poland: aflibercept, ranibizumab and brolucizumab. The fourth active substance, bevacizumab, can be prescribed off-label. All of them should be taken according to a similar regimen: three saturation injections given at monthly intervals. Intervals may be prolonged depending on the response to treatment $[7,8]$. Three different treatment regimens are currently used worldwide: fixed treatment regimen with monthly injections, pro re nata (PRN) injections according to the disease activity and treat-and-extend (T\&E) regimen, in which dose intervals are extended until macular neovascularization (MNV) is no longer active.

In the context of achieving endpoints such as improved best corrected visual acuity (BCVA) and remission of retinal edema expressed by decreased central retinal thickness (CRT), monthly injections are the most optimal treatment regimen; however, due to issues of safety, possible higher risk of geographic atrophy, optimization of costs, and patients' compliance, researchers have developed two more treatment regimens $[9,10]$.

\section{OPTICAL COHERENCE TOMOGRAPHY OF THE MACULA - WHAT CAN BE IMPROVED?}

The most important part of wAMD diagnostics and treatment response assessment is central retina monitoring: intraretinal and subretinal fluid accumulation in macular edema, MNV, and, in case unfavorable to the patient: enlarged areas of photoreceptor atrophy, subretinal scarring. Anatomical response assessment involves monitoring these characteristics.

Currently, optical coherence tomography (OCT) is a basic diagnostic tool for detecting all forms of macular disease. OCT is routinely used to diagnose and monitor AMD treatment. Scanning all retinal layers allows for visualization of all changes associated with the dis- ease at a very early stage, which is crucial for treatment implementation and, consequently, disease prognosis. During therapy monitoring, it is important to compare test results from subsequent checkpoints that will allow making decision about treatment continuation and scheduling the next follow-up visit or injection. These decisions are extremely important from the physician's and patient's point of view, so it is extremely important how they are made. Ideally, comparison of the test results should be precise, fast, and allow the patient to easily see the treatment progress and the reason for making any changes in the regimen. Typically, two scans are compared: performed at the current and previous visits, including the analysis of changes in the central macular thickness and a central scan preview (fig. 1).

\section{FIGURE 1}

\section{Study analysis based on the Macular Change protocol.}

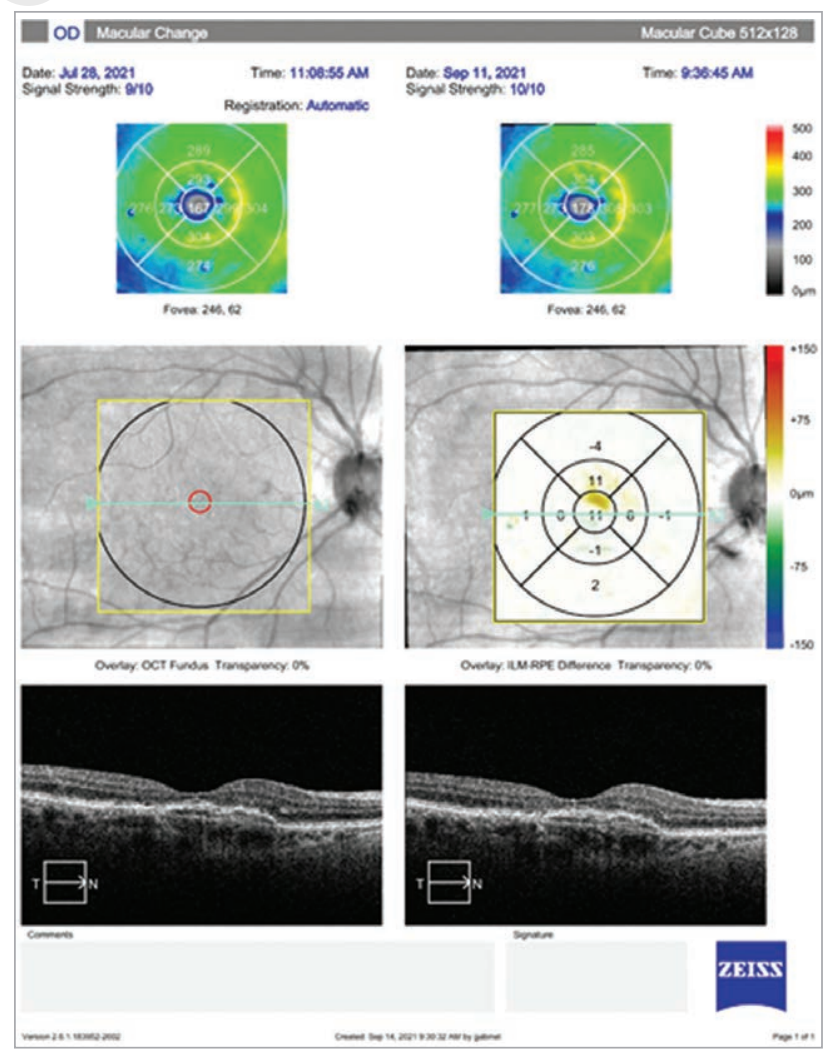

Therefore, it is difficult to make a binding decision, so for a more accurate assessment, the attending ophthalmologist is forced to manually analyze the scans from the subsequent examinations, which takes some time. If we add to this comparing injection dates to assess treatment effectiveness, the whole process is extremely time-consuming, although until recently it would seem that this is the only way to accurately analyze the condition of the 
macula and response to the chosen treatment regimen. Fortunately, modern technology comes to our aid.

\section{FORUM OPHTHALMICUM - OPERATING PRINCIPLES AND FUNCTIONALITY}

In our clinic, we use Forum ${ }^{\circ}$ by ZEISS. Forum is scalable ophthalmic software that, according to the manufacturer's definition, is a "data integrating and work efficiency system", and a tool supporting integration and advanced analysis of patient data for a practicing doctor. In other words, opening one program enables access to tests (macular and disc OCT, biometrics, field of view, fundus photography, OCT angiography) performed on a given patient on different devices. It also allows for comparison of test results, the addition of treatment regimens, and other medical events occurring throughout the entire observation period. We present below several Forum $^{\odot}$ features based on managing patients with AMD, however, that it is only part of this platform's capabilities. Firstly, after pressing the Macular Thickness Analysis button we get a preview of up to any three selected scans over time, instead of laborious analysis of individual tests. This allows for choosing choose dates of compared tests and analyzed numerical parameters or image types (cross-sectional B, HD, radial or en-face scans at different retina levels) (fig. 2).
In addition, to facilitate analysis of treatment regimen process, we can easily see at what point a patient received an injection. Select the drug that was used in a patient, the date and the type of medical event. We can choose from injection, topical medicine, or surgery. Our own event can also be defined. Each injection can be described manually by adding a comment, or by making a template, which will additionally speed up this procedure (fig. 3).

\section{FIGURE $(3$}

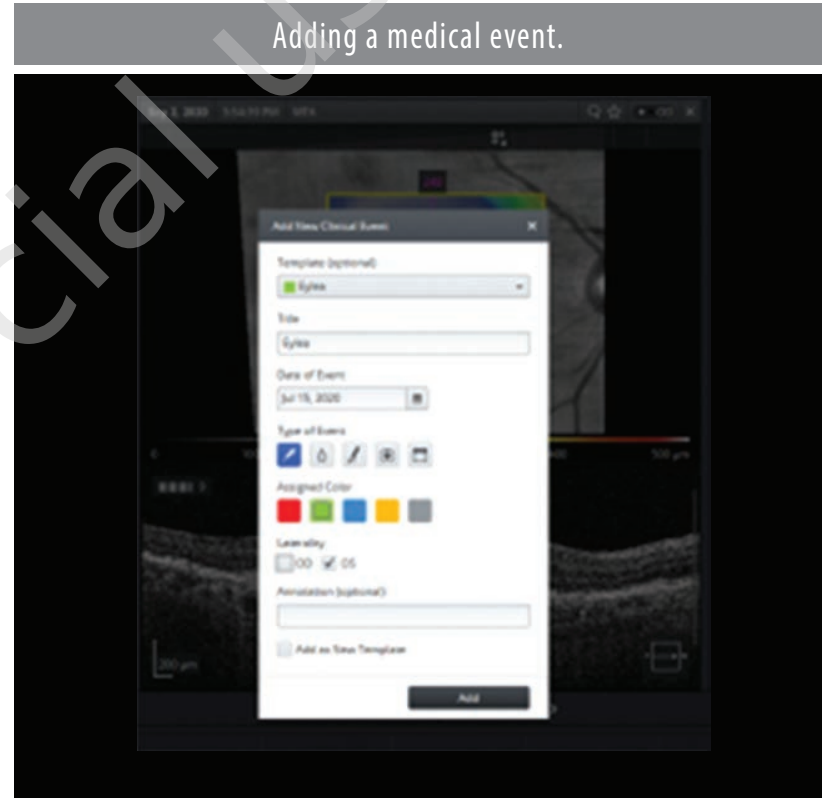

\section{FIGURE ( 2}

\section{Doctor-generated Forum ${ }^{\oplus}$ report.}

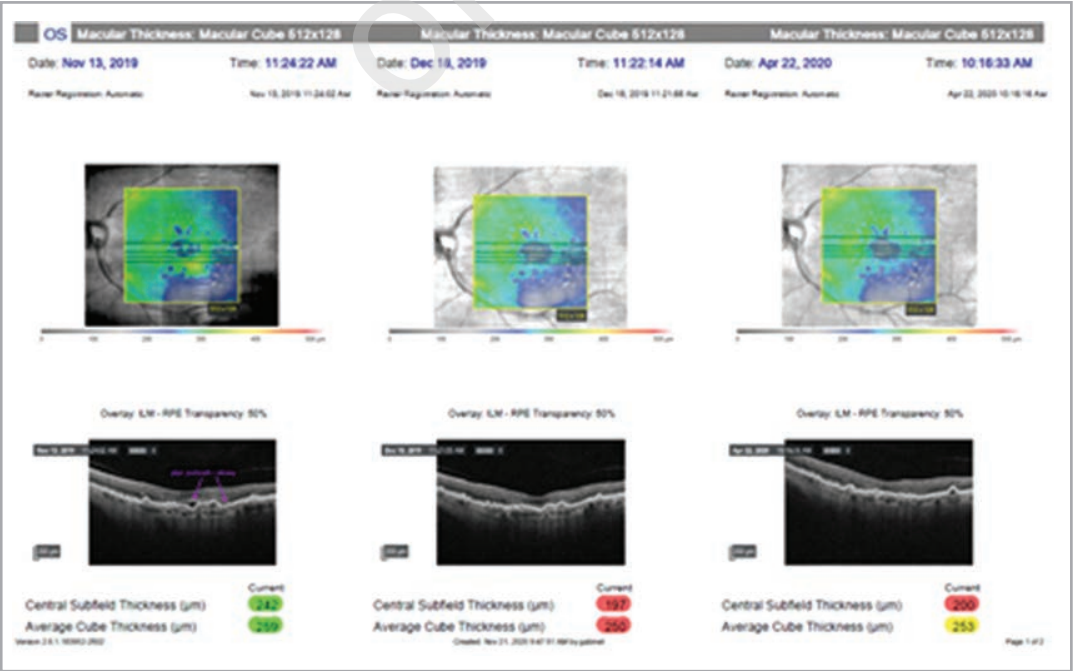


On the Macular Thickness Analysis tab, we can preview up to three recent tests (or others selected using the drag-and-drop method), and clearly see the course of the treatment so far (fig. 4).

Therapy regimens are becoming more and more popular, especially in the times of the current need to minimize visits and to plan them more precisely. This is known as "treat and extend" and is associated with extending or shortening the intervals between visits/injections depending on the condition of the macula. When we add data on the time of injection, we will receive information about the time elapsed between injections in Forum ${ }^{\bullet}$, by mousing over the date of a checkpoint. Depending on the condition of the macula, this allows us to precisely plan the date of the next drug administration (fig. 5).

We can also set an injection template in which different active substances are marked with different colors (fig. 6). This is especially useful in a so-called switch, when we decide to change the administered drug for various reasons (most often a lack of satisfactory effectiveness or risk factors). When analyzing the course of treatment we can immediately see when the change took place and what drugs were used (fig. 7).
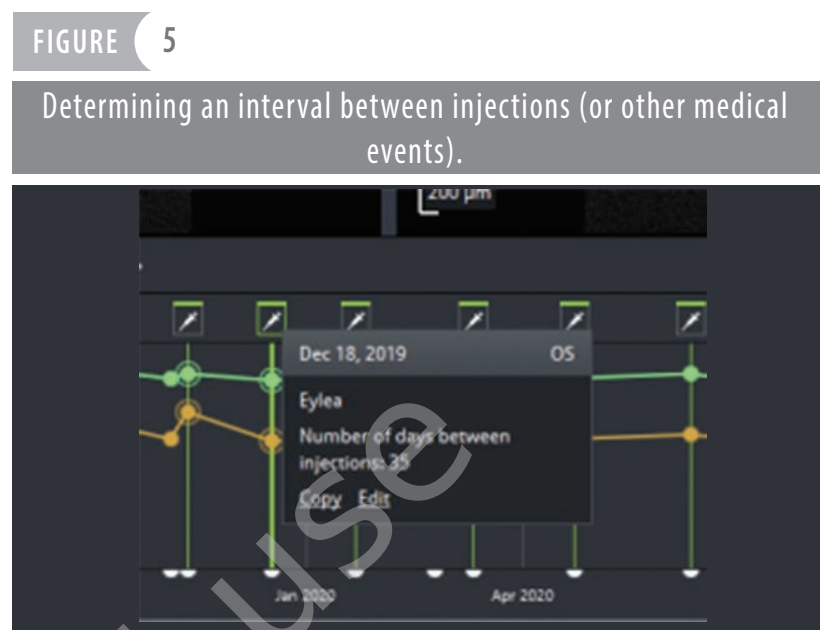

\section{FIGURE}

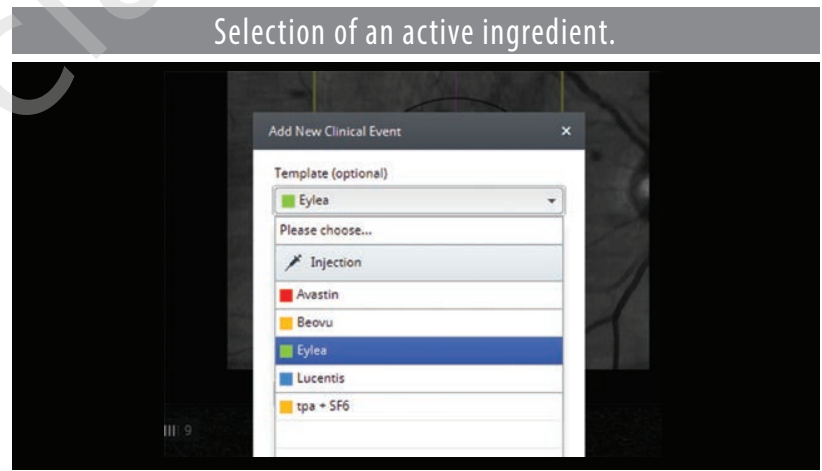

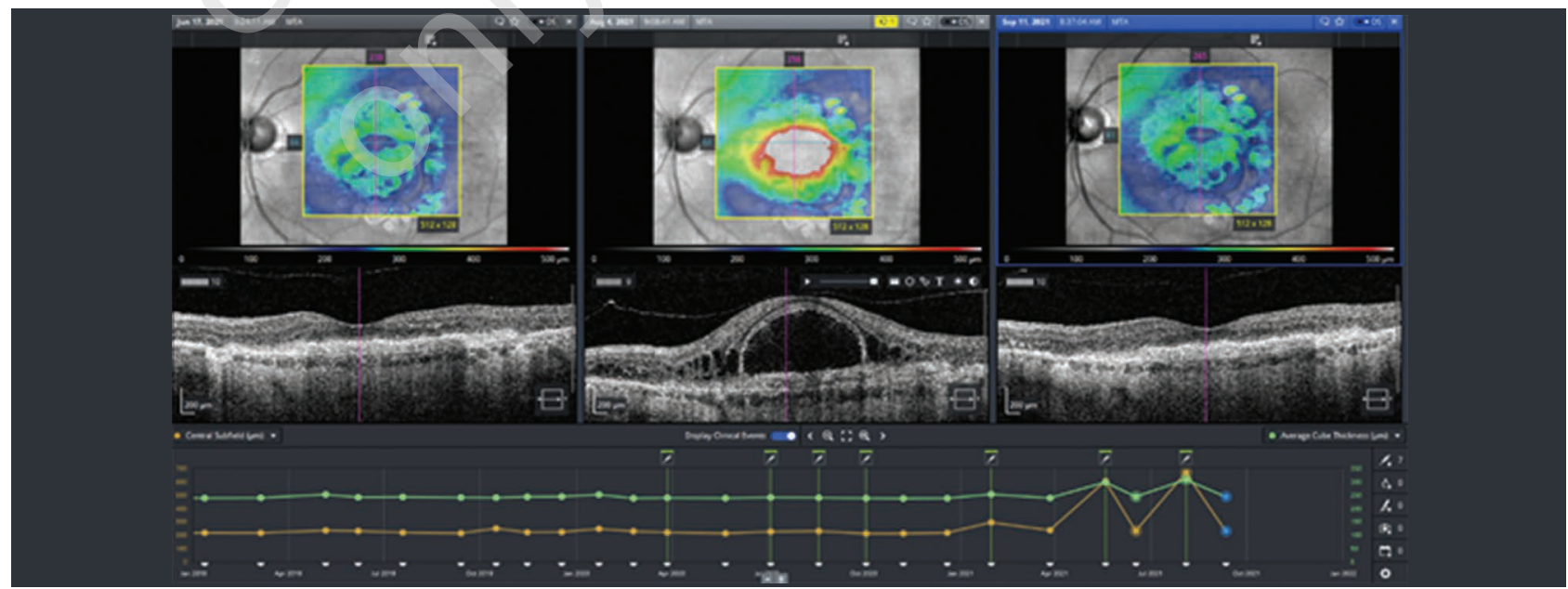


A switch. The green color indicates injection of aflibercept, and blue of ranibizumab.

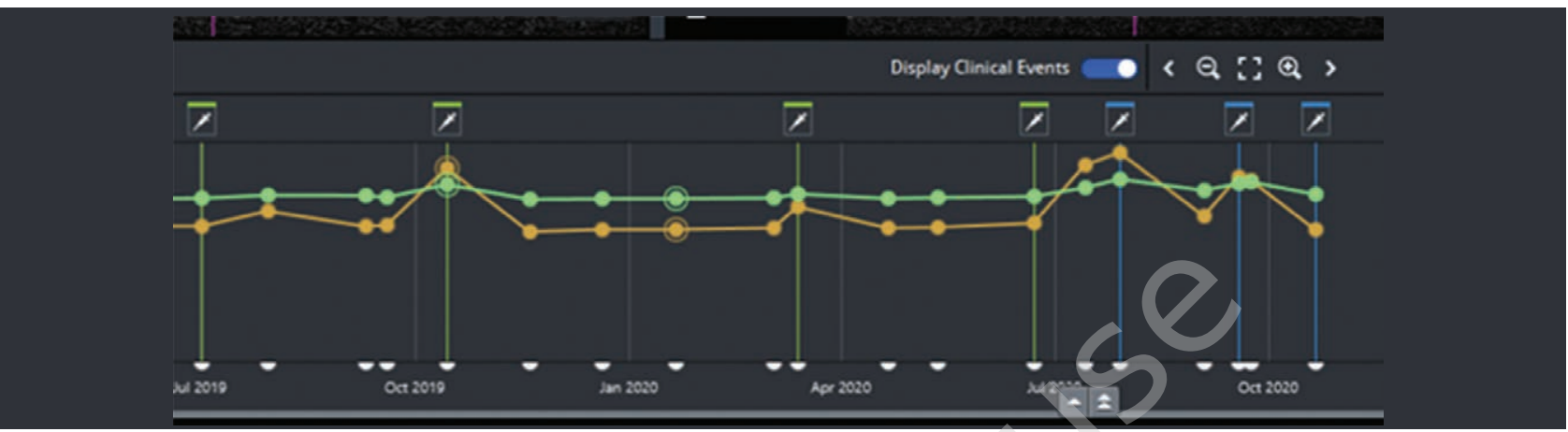

The ideal analytical test instrument mainly facilitates the doctor to make the right therapeutic decisions, however, communication with the patient is an equally important aspect of our work. The Forum ${ }^{\circledR}$ software can also be used to easily show the patient the course of their disease and illustrate the changes that have occurred over time.

Figure 8 shows that by selecting the en-face scans option at different levels of the retina, including the choroid, we can clearly illustrate the enlargement of the central atrophy area associated with irreversible and untreatable visual impairment. It is said that a picture is worth more than a thousand words. And this seems to be the case here.

\section{CONCLUSIONS}

Using Forum ${ }^{\circ}$ speeds up the diagnostic process, facilitates effective analysis of the treatment regimen, and allows for easier communication with the patient. After working closely with this device, the process becomes easy and facilitates work of doctors treating macular diseases, and many more. This will be followed in the next section.

Figures: from the author's own materials.

\section{Progression of foveal atrophy - despite a reduction of swelling as a result of the treatment, the patient complained of progressive deterioration in the quality of vision.}

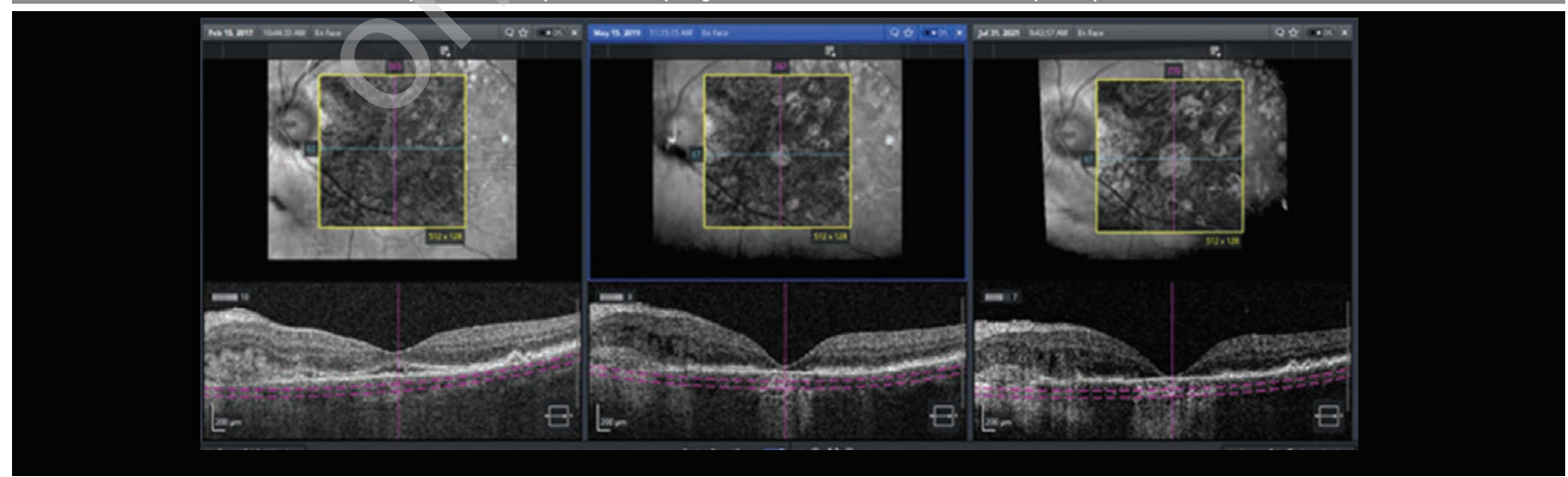




\section{CORRESPONDENCE}

Dorota Maria Kaczmarek, MD, PhD

Clinical Ophthalmology Center Spektrum

53-334 Wrocław, ul. Zaolziańska 4

e-mail: dorota.kaczmarek@spektrum.wroc.pl

\section{ORCID}

Dorota Maria Kaczmarek - ID - http://orcid.org/0000-0002-0397-1115

\section{References}

1. Chakravarthy U, Wong TY, Fletcher A et al. 'Clinical risk factors for age-related macular degeneration: a systematic review and meta-analysis. BMC Ophthalmol. 2010; 10: 31. http://doi.org/10.1186/1471-2415-10-31.

2. Bhutto I, Lutty G. Understanding age-related macular degeneration (AMD): Relationships between the photoreceptor/retinal pigment epithelium/Bruch's membrane/choriocapillaris complex. Mol Aspects Med. 2012; 33(4): 295-317. http://doi.org/10.1016/j. mam.2012.04.005.

3. Jager RD, Mieler WF, Miller JW. Age-Related Macular Degeneration. N Engl J Med. 2008; 358(24): 2606-17. http://doi.org/10.1056/NEJMra0801537.

4. Chakravarthy U, Evans J, Rosenfeld PJ. Age related macular degeneration. BMJ. 2010; 340: c981.

5. Wong WL, Su X, Li X et al. Global prevalence of age-related macular degeneration and disease burden projection for 2020 and 2040 : a systematic review and meta-analysis. Lancet Glob Health. 2014; 2(2): e106-16. http://doi.org/10.1016/S2214-109X(13)70145-1.

6. Spaide RF, Jaffe GJ, Sarraf D et al. Consensus Nomenclature for Reporting Neovascular Age-Related Macular Degeneration Data. Ophthalmology. 2020; 127(5): 616-36. http://doi.org/10.1016/j.ophtha.2019.11.004.

7. Charakterystyka produktu leczniczego: Bayer Pharma AG, Eylea aflibercept.

8. Charakterystyka produktu leczniczego: Novartis, Lucentis ranibizumab.

9. Androudi S, Dastiridou A, Pharmakakis N et al. Guidelines for the Management of Wet Age-Related Macular Degeneration: Recommendations from a Panel of Greek Experts. Adv Ther. 2016; 33: 715-26. http://doi.org/10.1007/s12325-016-0332-7.

10. Treat-and-Extend Strategy: Is There a Consensus? American Academy of Ophthalmology 2016. https://www.aao.org/eyenet/article/ treat-extend-strategy-is-there-consensus (access: 5.02.2019).

Conflict of interest:

None.

Financial support:

None.

Ethics:

The content presented in the article complies with the principles of the Helsinki

Declaration, EU directives and harmonized requirements for biomedical journals. 